Check for updates

Cite this: RSC Adv., 2017, 7, 21342

Received 20th February 2017

Accepted 8th April 2017

DOI: 10.1039/c7ra02113e

rsc.li/rsc-advances

\section{Melatonin enhances the anti-tumor effect of sorafenib via AKT/p27-mediated cell cycle arrest in hepatocarcinoma cell lines $\uparrow$}

\author{
Fei Long, $\dot{\dagger}^{\mathrm{a}}$ Chengyong Dong, $\ddagger^{\mathrm{a}}$ Keqiu Jiang, ${ }^{a}$ Yakun $\mathrm{Xu},{ }^{a}$ Xinming Chi, \\ Deguang Sun, ${ }^{a}$ Rui Liang, ${ }^{a}$ Zhenming Gao, ${ }^{a}$ Shujuan Shao*b and Liming Wang (DD *a
}

\begin{abstract}
Hepatocellular carcinoma (HCC) is the fifth most common cancer worldwide, often diagnosed in late stages when most therapeutic methods are not very effective. The introduction of the multikinase inhibitor sorafenib as the standard of care has opened a window of hope for patients with advanced HCC, patients with very poor prognosis; however, patients usually develop acquired resistance to sorafenib limiting its therapeutic benefits. Melatonin (MT), an indoleamine compound produced in the pineal gland, has shown a substantial beneficial effect in increasing the efficacy of common anticancer drugs and decreasing their toxic effects. Here we demonstrate that MT potentiated the sorafenib-mediated inhibition of cell viability and colony formation in HCC cell lines. Moreover, combined treatment of MT and sorafenib enhanced the cell cycle arrest of HCC cells at the G0/G1 phase. Co-treatment of sorafenib and MT was found to upregulate p27, an inhibitor of several cyclin-dependent kinases (CDK), and downregulate $\mathrm{p}-\mathrm{AKT}$, c-myc, cyclin D1 and CDK4/6 protein expression. Furthermore, overexpression of p-AKT using SC79 reversed the effect of sorafenib and MT combination on cell viability and growth of HCC cells. These results suggest that the AKT pathway might be critical for the enhanced anticancer effect observed after co-treatment with MT and sorafenib. Taken together, our findings demonstrated that AKT/p27-mediated cell growth arrest induced by MT increased the sensitivity of HCC cells to the effect of sorafenib.
\end{abstract}

\section{Introduction}

Hepatocellular carcinoma (HCC) is one of the most common malignant tumors worldwide with approximately more than half a million new cases diagnosed annually. ${ }^{1}$ HCC is highly resistant to chemotherapeutic agents. Indeed, there is a lack of efficient chemotherapy drugs, leaving local ablation, surgical resection, and liver transplantation as the only therapeutic options for patients diagnosed with early-stage HCC. Unfortunately, the disease is often diagnosed in late stages when most patients are not suitable for local ablation or surgery. The introduction of the multikinase inhibitor sorafenib as the standard of care has opened a window of hope for advanced HCC patients with very poor prognosis. ${ }^{2}$ Sorafenib has been shown to successfully inhibit tumor cell proliferation and

\footnotetext{
${ }^{a}$ Division of Hepatobiliary and Pancreatic Surgery, Department of General Surgery, The Second Affiliated Hospital of Dalian Medical University, Dalian, Liaoning, China. E-mail: wangbcc259@163.com; Fax: +86-0411-84671291; Tel: +86-0411-84671291

${ }^{b}$ Key Laboratory of Proteomics, Dalian Medical University, Dalian, China. E-mail: shaoshujuan2006@126.com; Fax: +86-0411-86110314; Tel: +86-0411-86110314

$\uparrow$ Electronic supplementary information (ESI) available. See DOI: 10.1039/c7ra02113e

\$ These authors have contributed equally to this work.
}

vasculogenesis by targeting RAF/MEK/ERK, as well as JAK/STAT pathways. ${ }^{3,4}$ However, the low rate of tumor response, most likely due to primary and acquired drug resistance, has limited the overall therapeutic effect of sorafenib on HCC patients. ${ }^{5,6}$ In addition, many patients have multiple undesirable side effects. ${ }^{7}$ Thus, a synergistic combinatorial approach that includes sorafenib at low doses could be a better therapeutic strategy in HCC to improve the anti-tumor effect of sorafenib and, at the same time, decrease its toxicity.

Although synthesis of melatonin (MT) occurs in multiple organs, the major site of MT synthesis is the pineal gland. At physiological concentrations, MT functions as a major regulator of the circadian rhythms and sleep. ${ }^{8,9}$ Moreover, it has been shown to regulate obesity, ${ }^{10}$ the immune systems ${ }^{11}$ and oncostasis. ${ }^{12-14}$

In addition, previous studies have also shown MT plays a role in tumor growth and gene regulation in cancer cells. ${ }^{15,16}$ MT inhibited cell proliferation of human B-lymphoma cells, ${ }^{17}$ human myeloid leukemia cells HL-60 ${ }^{18}$ and human neuroblastoma cancer cells. ${ }^{19}$ Furthermore, MT exerts a protective function by preventing cell damage, ${ }^{20-22}$ particularly in neural cells..$^{23,24}$ A plethora of evidence has unequivocally demonstrated that MT decreases the toxicity associated with chemotherapeutic agents both in animals as well as in patients. ${ }^{25,26}$ 
In this study, we aim to investigate the beneficial impact of combining MT to sorafenib, the standard of care, in HCC and the mechanism by which MT improves the anti-tumor effect of sorafenib.

\section{Experimental}

\subsection{Cell lines and cell culture}

Human HCC cell lines Bel7402, SMMC-7721 and the normal hepatocyte cell lines L02 were purchased from KeyGen Biotech Company (Nanjing, China). Cell lines were cultured in RPMI Medium 1640 (Gibco, Gaithersburg, MD, USA), supplemented with $10 \%$ fetal bovine serum (FBS) (PAN BIOTECH, Aidenbach, Germany) and 1\% penicillin/streptomycin (100 $\mathrm{U} \mathrm{ml}^{-1}$, Gibco). Cells were incubated in a humidified atmosphere containing $5 \% \mathrm{CO}_{2}$ at $37{ }^{\circ} \mathrm{C}$.

\subsection{Reagents and antibodies}

MT was obtained from Sigma (St. Louis, MO, USA) and was dissolved in an appropriate amount of DMSO. Sorafenib and SC79 were purchased from Selleckchem (Houston, TX, USA) and were also dissolved in an appropriate amount of DMSO. The above three reagents were added to the complete cell culture medium to attain the stipulated concentration. The maximum concentration of DMSO in media was $0.1 \%$ and the concentration of DMSO in the control groups was $0.1 \%$ constantly. The Cell Counting Kit-8 (CCK8) was purchased from Dojindo (Kumamoto, Japan). Rabbit polyclonal antibodies for total AKT, phospho-AKT (p-AKT) (Ser473), total ERK, phospho-ERK (p-ERK) (Thr202/Tyr204), p21, cyclin D1, phospho-Rb (p-Rb) (Ser807/811) and $\beta$-actin were purchased from Cell Signaling (Danvers, MA, USA). Rabbit polyclonal antibodies for p27, CDK4, CDK6, cyclin E1, cyclin A1 and c-myc were obtained from Protein Tech (Chicago, IL, USA). All the secondary antibodies were purchased from Protein Tech (Chicago, IL, USA).

\subsection{Cell viability assay}

Cell viability was tested using the CCK-8 assay in accordance with the manufacturer's instructions. Hepatocellular carcinoma (HCC) cells $\left(2 \times 10^{3}\right)$ were seeded into 96-well plates and cultured in $100 \mu \mathrm{l}$ of cell culture medium. After 24 hours, cells were treated with MT and/or sorafenib at the indicated concentrations for 48 or 72 hours (in the presence or absence of AKT activator SC79 $\left[8.0 \mu \mathrm{g} \mathrm{ml}{ }^{-1}\right]$ for 48 hours). Then, the medium was replaced by $100 \mu \mathrm{l}$ of a mixture of cell culture medium $(90 \mu \mathrm{l})$ and CCK-8 reagent $(10 \mu \mathrm{l})$ and cells were incubated at $37{ }^{\circ} \mathrm{C}$ or 2 hours. Finally, the optical density was determined at a wavelength of $450 \mathrm{~nm}$ by an EnSpireTM 2300 Multilabel Reader (PerkinElmer, Waltham, MA, USA). Three replicates were measured for each test. At least three independent experiments were performed.

\subsection{Clonogenic survival assay}

Cells were seeded into a 6-well culture dish in quadruplicate (800 cells per well). After 48 hours of incubation, cultures were replaced with fresh medium containing MT and/or sorafenib in the indicated concentrations for 2 weeks. During this time the medium was renewed every 4 days. After 2 weeks, cell colonies were washed with phosphate buffered saline (PBS) buffer for three times, fixed in paraformaldehyde (PFA) for 15 minutes, and stained using crystal violet (Sigma-Aldrich, St. Louis, MO, USA) for 15 minutes at room temperature. Clones were visually counted and colony formation efficiency was calculated.

\subsection{Cell cycle analysis}

Cells were seeded into a 6-well culture dish. After 24 hours of incubation, cultures were replaced by fresh medium containing MT and/or sorafenib at the indicated concentrations for 48 hours (in the presence or absence of AKT activator SC79 [8.0 $\mu \mathrm{g}$ $\mathrm{ml}^{-1}$ ] for 48 hours) and cells were harvested by trypsinization, and, then, fixed overnight at $4{ }^{\circ} \mathrm{C}$ with $75 \%$ cold ethanol. Fixed cells were treated with $100 \mu \mathrm{l}$ of RNase $\mathrm{A}\left(10 \mu \mathrm{g} \mathrm{ml}{ }^{-1}\right)$ at $37^{\circ} \mathrm{C}$ for 30 minutes and stained with $400 \mu \mathrm{l}$ of PI $\left(50 \mu \mathrm{g} \mathrm{ml}{ }^{-1}\right)$ at room temperature in dark conditions for 30 minutes. The cell cycle profiles were obtained using a BD FacsCalibur (BD, Franklin Lanes, NJ, USA) flow cytometer.

\subsection{Western blot analysis}

Cells were cultured in $10 \mathrm{~cm}$-diameter dishes and treated either with DMSO, single agent or combination (in the presence or absence of AKT activator SC79 $\left[8.0 \mu \mathrm{g} \mathrm{ml}^{-1}\right]$ ) for appropriate timepoints. Western blot analyses were performed using standard procedures. Actin was used as a loading control. The signal was detected using secondary antibodies coupled with HRP (Jackson laboratory). The luminescent signal was detected using a LAS3000 Luminescent Image analyzer (Fujifilm, Valhalla, NY).

\subsection{Animal study}

$\mathrm{BALB} / \mathrm{c}$ nude female mice ( 4 weeks of age) were used in this study and were purchased from the Dalian Medical University SPF Laboratory Animal Center (Dalian, China). Mice, weighing between 13 and $18 \mathrm{~g}$, were fed in a specific pathogen-free area. To evaluate the therapeutic efficacy of MT and sorafenib in human HCC cell lines SMMC-7721 using a murine model, SMMC-7721 cells $\left(5 \times 10^{6}\right.$ in $100 \mu \mathrm{l}$ PBS $)$ were injected subcutaneously near the axillary fossa of nude mice. After two weeks, when tumor diameter reached $3 \mathrm{~mm} \times 4 \mathrm{~mm}$, tumor cell-inoculated mice were randomly divided into four treatment groups, each containing four mice. Group A, treated with normal saline (NS); group B treated with $25 \mathrm{mg} \mathrm{kg}^{-1} \mathrm{MT}$ (i.p.); group C treated with $10 \mathrm{mg} \mathrm{kg}^{-1}$ sorafenib (orally); group D treated with MT (i.p.) and sorafenib (orally) and each group was administrated every day. Tumors were measured using a caliper every 3 days and the formula $V=1 / 2$ (width ${ }^{2} \times$ length) was used to calculate the tumor volume. The weights of the mice were also recorded every 3 days. On day 32 after cell inoculation, all mice were sacrificed with ether anesthesia and tumor weight in each mouse was measured.

All experiments were performed in compliance with the recommendations established by the Animal Care and Ethics Committee of Dalian Medical University. The protocol was approved by the Animal Care and Ethics Committee of Dalian Medical University. 


\subsection{Statistical analysis}

All experiments were repeated at least three times. Means and standard deviations were calculated from at least three different measurements. GraphPad Prism software was used for statistical analysis. Statistical comparisons were implemented using one-way ANOVA test. A $p$-value $<0.05$ was considered to be statistically significant. SPSS v13.0 software was applied for all statistical analyses.

\section{Results}

3.1 Co-treatment of MT and sorafenib decreases the cell viability and alters morphology in HCC cell lines

To determine whether MT could potentiate the sorafenibinduced inhibition of hepatoma cell lines survival, we first investigated the effect of MT or sorafenib alone on cell viability in HCC cell lines Bel-7402 and SMMC-7721 using a CCK-8 assay. Treatments with MT alone [200-2000 $\mu \mathrm{M}$ ] or sorafenib alone [1$12 \mu \mathrm{M}$ ] for 48 or 72 hours have shown to considerably suppress hepatoma cell viability in a dose and time-dependent manner (Fig. 1A and B). Combined treatment of MT [1.0 mM] and sorafenib $\left[\begin{array}{ll}4-8 & \mu \mathrm{M}\end{array}\right]$ for 48 hours significantly enhanced the sorafenib-mediated inhibition of cell viability in both Bel-7402 and SMMC-7721 cells as compared with those treated with either MT or sorafenib alone (Fig. 1C). Additionally, we analyzed the changes in cell spreading and morphology of Bel-7402 and SMMC-7721 cells cultured in 6-well plates (Fig. 1D; $8 \mu \mathrm{M}$ sorafenib and/or $1 \mathrm{mM}$ MT for Bel-7402; $4 \mu \mathrm{M}$ sorafenib and/or $1 \mathrm{mM}$ MT for SMMC-7721). The combined treatment showed a significant reduction in cell density as well as changes in cell morphology, where cells become smaller and granulated in

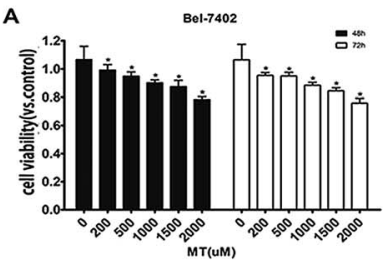

B

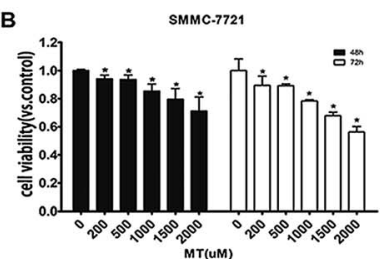

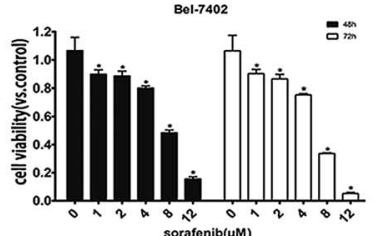

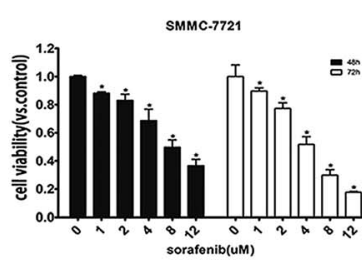

C

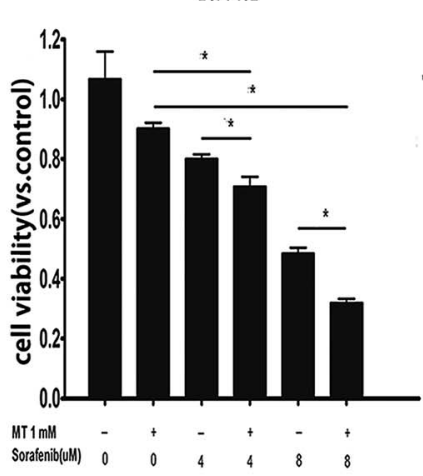

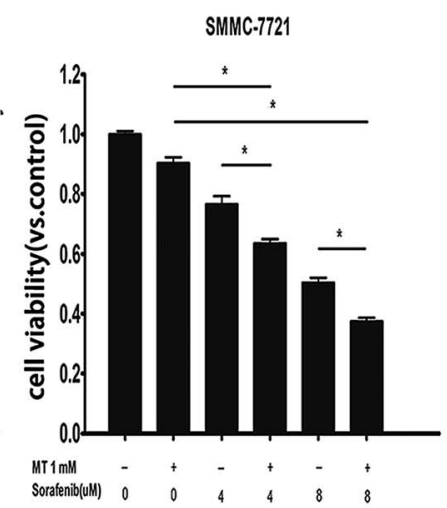

L02

E

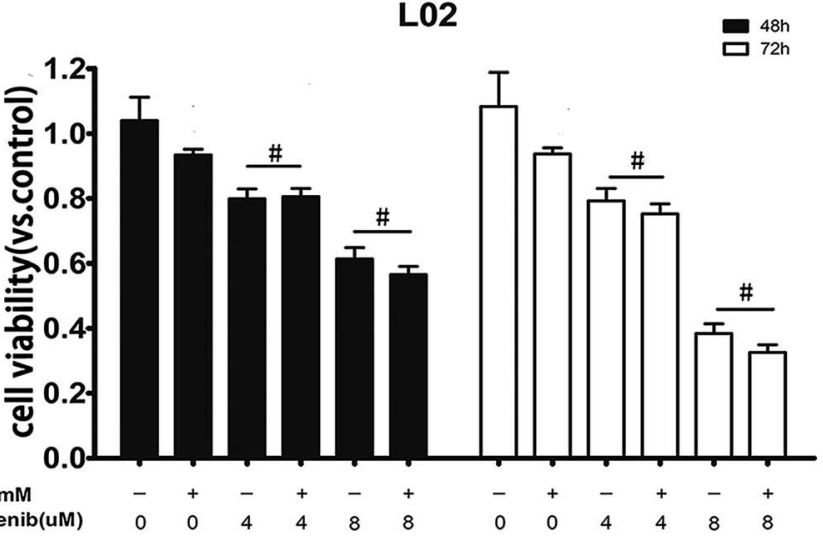

Fig. 1 Melatonin (MT) potentiated the inhibitory effect of sorafenib on the viability of hepatocarcinoma cells but does not affect normal liver cells. (A and B) Bel-7402 and SMMC-7721 cells were treated with MT or sorafenib from 0-72 hours at the indicated doses. 48 and 72 hours after treatment, cell viability was determined using a CCK- 8 assay. Cells treated with vehicle (DMSO) were set as $100 \%$ cell viability. The percentage of cell viability in each treatment group was calculated relative to cells treated with vehicle control. (C) Bel-7402 and SMMC-7721 cells were treated with MT or/and sorafenib during 48 hours at the indicated doses. 48 hours after treatment, cell viability was determined using a CCK- 8 assay. (D) Changes in cell morphology and spreading of Bel-7402 and SMMC-7721 cells treated with sorafenib [8 $\mu$ M] for Bel-7402 and [4 $\mu$ M] for SMMC7721 and/or MT [1 mM] for 48 hours in 6-well plates were observed and photographed using an Olympus inverted microscope with digital camera. Each cell line was photographed with a magnification of $4 \times 10$ fold (the picture above) and $20 \times 10$ fold (the picture below) (E) LO2 cells (a normal hepatocyte cell lines) were treated with MT or/and sorafenib from 0-72 hours at the indicated doses. After 48 and 72 hours after treatment, cell viability was determined using a CCK- 8 assay. The data are presented as mean \pm SD of three separate experiments. $P<0.05$ indicates significant differences between treatment groups and control groups or among treated groups. \#P>0.05 represents no significant differences among groups. 
shape when compared with those treated either with sorafenib or MT alone. These results demonstrated that combined treatment of sorafenib and MT induces changes in cell spreading and morphology of hepatoma cells.

It has been reported that MT exerts a protective effect in normal cells. ${ }^{27,28}$ Therefore, we investigated whether cotreatment of MT and sorafenib had an impact on normal L02 cells. Treatment with sorafenib $\left[\begin{array}{lll}4-8 & \mu \mathrm{M}\end{array}\right]$ for $48-72$ hours significantly reduced cell viability compared with untreated controls (Fig. 1E). Interestingly, in contrast to hepatocarcinoma cells, MT had no significant effect on cell viability when it is combined to sorafenib compared to single drug treatment.

\subsection{MT potentiates the inhibitory effect of sorafenib on the colony formation of hepatocarcinoma cells}

To further confirm the effect of MT in enhancing the sorafenibmediated inhibition of cell growth in HCC cell lines. We employed a colony formation assay where Bel-7402 and SMMC7721 cells were treated with MT and/or sorafenib at specific concentrations for 2 weeks. Both MT and sorafenib caused an observable decrease in the numbers of colonies in both cell lines (Fig. 2A and B). Dramatically, the combination of MT and sorafenib triggered a more pronounced inhibition of colony formation compared to single treatment.

\subsection{MT enhances the antitumor effect of sorafenib via G0/G1 phase arrest}

To explore whether MT could enhance the antitumor effect of sorafenib in Bel-7402 cells through the alteration of the cell cycle, we examined cell cycle progression using FACS analysis. MT or sorafenib arrested the cell cycle at the G0/G1 phase in Bel7402 cells compared with the control groups. However, combined treatment of MT and sorafenib further enhanced cell cycle arrest at G0/G1 phase compared with single treatment with sorafenib (Fig. 3A and B).

Alterations in the checkpoint mechanisms of the cell cycle lead to cell proliferation. The Cyclin D1/CDK4/6 complex increases the phosphorylation of $\mathrm{Rb}(\mathrm{p}-\mathrm{Rb})$ followed by cell entry into the $\mathrm{S}$ phase from the G1 phase. ${ }^{29}$ Since cells were arrested at the G0/G1 phase, the protein expression of Cyclin D1, CDK4, CDK6, cyclin E1 and p-Rb, responsible for the transition from G0/G1 to $S$ phase were analyzed using western blot. As expected, Cyclin D1, CDK4, CDK6 and the downstream p-Rb
A

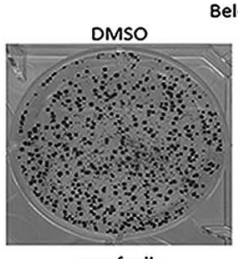

sorafenib
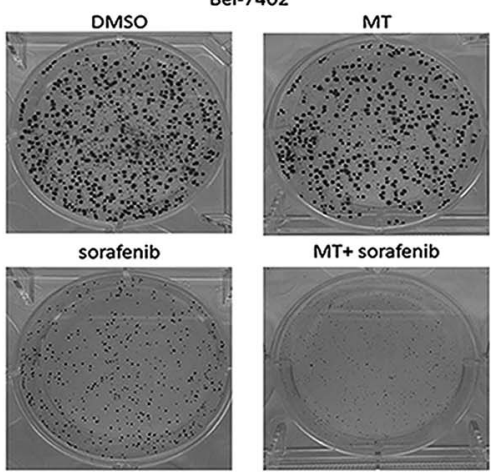

SMMC-7721

B

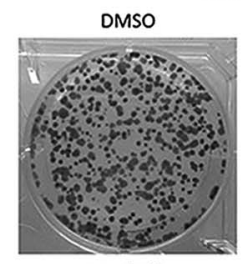

sorafenib

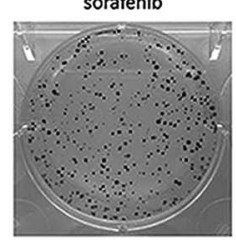

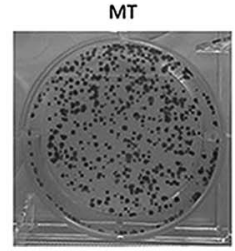

$\mathrm{MT}+$ sorafenib

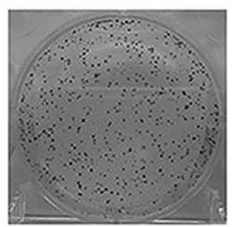

Bel-7402

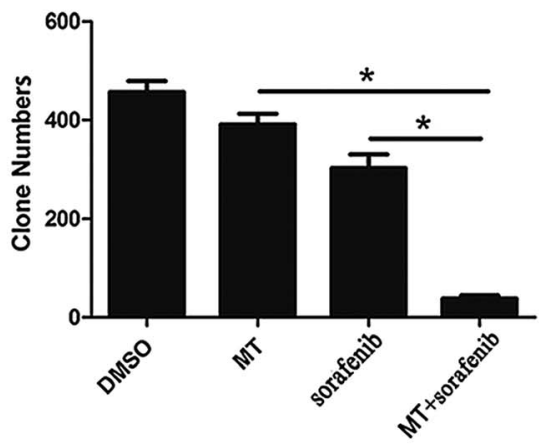

SMMC-7721

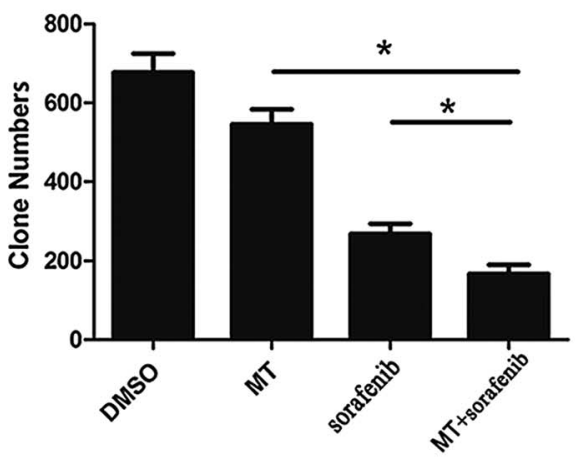

Fig. 2 MT potentiated the inhibitory effect of sorafenib on colony formation of hepatocarcinoma cells. (A) Bel-7402 cells were grown in 6 -well plates in quadruplicate $(800$ cells per well). After 48 hours, cultures were replaced with fresh medium containing MT [1 mM] and/or sorafenib [8 $\mu \mathrm{M}]$ for 2 weeks. After 2 weeks, the colonies were stained with $1 \%$ crystal violet and were counted. Clone numbers are shown in the bar graph (right panel). (B) SMMC-7721 cells were grown in 6-well plates in quadruplicate (800 cells per well). After 48 hours, cultures were replaced with fresh medium containing MT [1 mM] and/or sorafenib [4 $\mu \mathrm{M}]$ for 2 weeks. After 2 weeks, the colonies were stained with $1 \%$ crystal violet and were counted. Clone numbers are also shown in the bar graph (right panel). Bars represent mean \pm SD from 3 independent experiments ( $* P<0$. 05 indicates significant difference among the groups). 

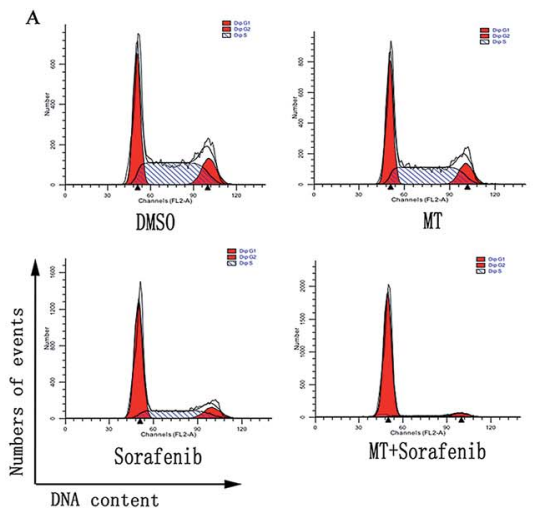

C

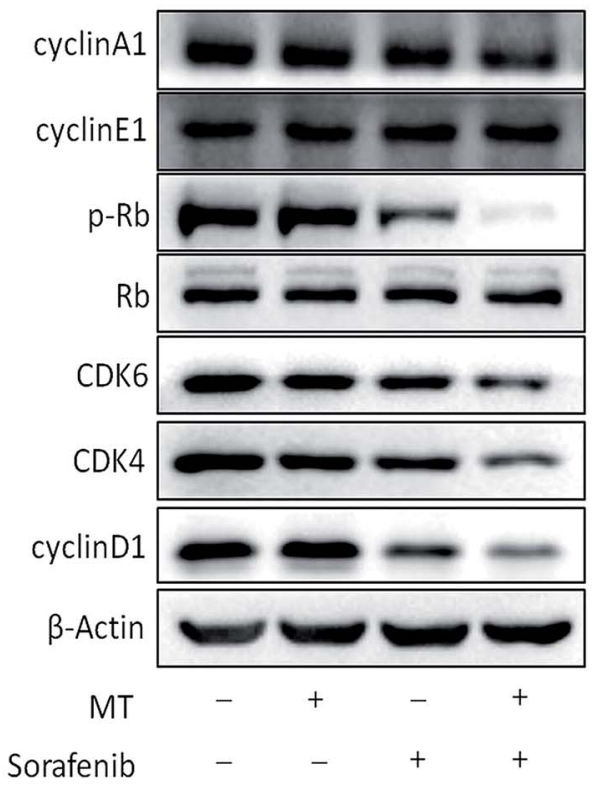

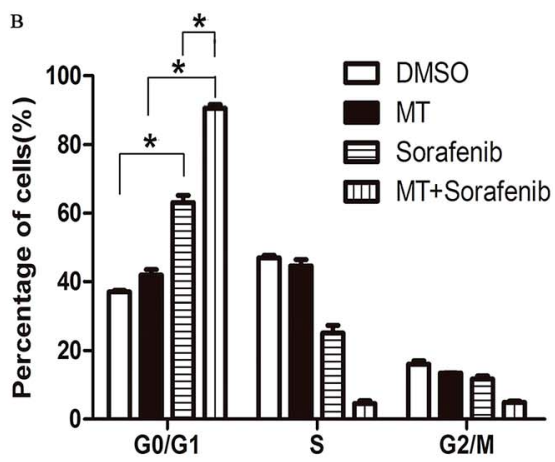

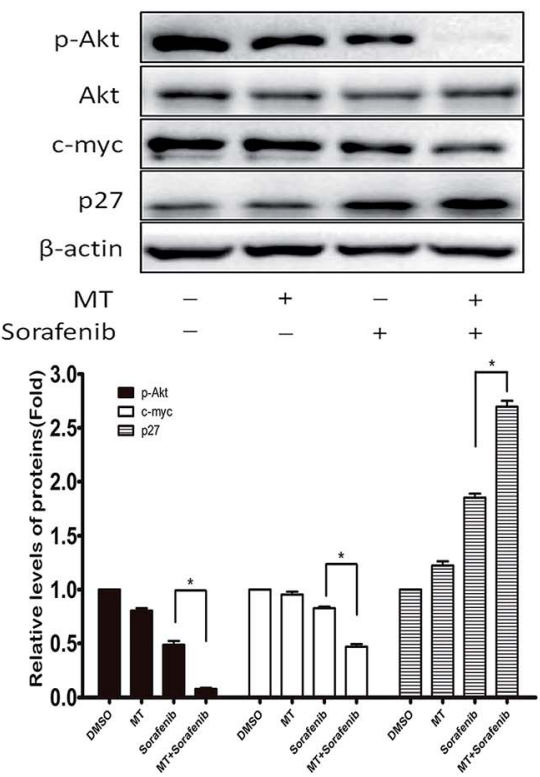

Fig. 3 MT enhanced the antitumor effects of sorafenib via G0/G1 phase arrest. Bel-7402 cells were treated with MT [1.0 mM] or/and sorafenib $[8.0 \mu \mathrm{M}]$ for 48 hours. (A and B) Flow cytometry was performed to analyse the progression of the cell cycle in Bel-7402. The percentage of cells $(\%)$ is shown in the bar graph. (C and D) Expression of cycle-related proteins and relevant regulatory proteins in cell lysates were detected using western blot. $(* P<0.05$ indicates significant difference among the groups).

were down-regulated following combined treatment of MT and sorafenib compared with the other groups, despite no changes were observed in Cyclin E1 and Cyclin A1 expression (Fig. 3C). Thus, these results demonstrated that the combined treatment of MT and sorafenib caused cell cycle arrest at the G0/G1 phase in Bel-7402 cells via downregulation of Cyclin D1, CDK4, CDK6, and $\mathrm{p}-\mathrm{Rb}$.

The p27Kip1 gene is located on chromosome $12 \mathrm{p} 13 .^{30}$ The CDK-inhibitory domain resides in the $\mathrm{N}$-terminal portion of p27Kip1 and is sufficient to arrest cells at G0/G1. ${ }^{31}$ In early G1, phosphorylation of p27Kip1 at Ser10 by AKT facilitates its binding to chromosomal maintenance 1 (CRM1) and its transport from the nucleus to the cytoplasm. ${ }^{32}$ Furthermore, in the early G1 phase, p27Kip1 phosphorylation at Thr157 and Thr198 by AKT causes a delay in nuclear import. ${ }^{33}$ MYC can also suppress p27Kip1 expression through several pathways. ${ }^{32}$ Thus, we examined the expression of p27, c-myc and AKT.
Co-treatment with MT and sorafenib downregulated p-AKT and c-myc and up-regulated p27 protein levels (Fig. 3D).

\subsection{Overexpression of p-AKT reverses the effect of MT and sorafenib co-treatment on cell viability in HCC cell lines}

To explore the impact of p-AKT on the growth of HCC cells, we assessed the effect of reactivation of p-AKT on MT and sorafenib-induced inhibition of cell viability. Interestingly, upregulation of p-AKT by SC79 $\left[8.0 \mu \mathrm{g} \mathrm{ml}^{-1}\right]$ abrogated the G0/ G1 phase arrest (Fig. 4A) and the inhibition of cell viability (Fig. 4B) induced by the combination of MT and sorafenib. In addition, the reactivation of $\mathrm{p}$-AKT reversed the combined treatment-induced increase of p27 and decrease of c-myc (Fig. 4C). Together, these results suggested that the inhibition of AKT pathway might be the underlying mechanism by which MT and sorafenib combination induces cell cycle arrest and enhances the cytotoxicity in HCC cells. 

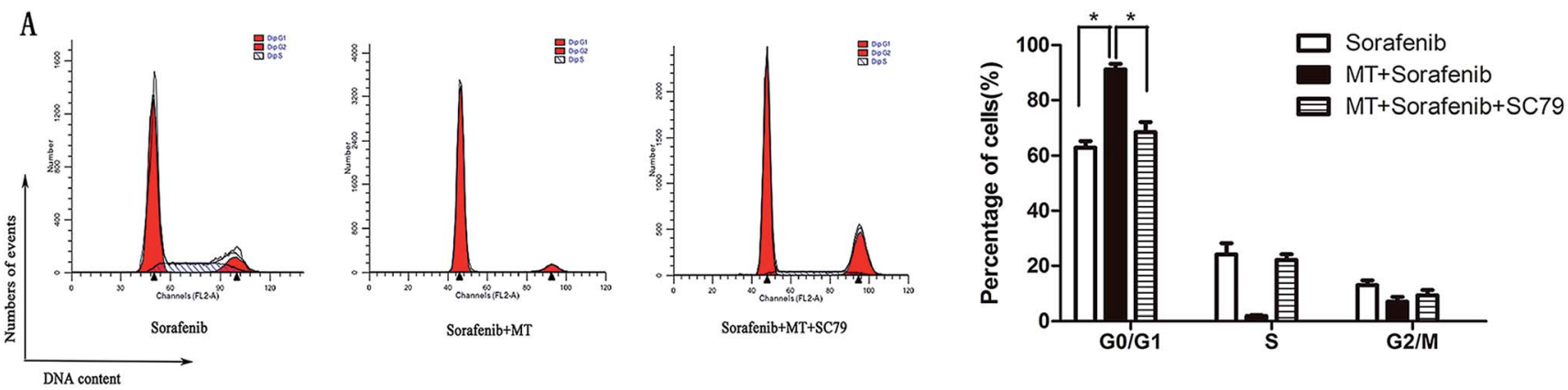

B

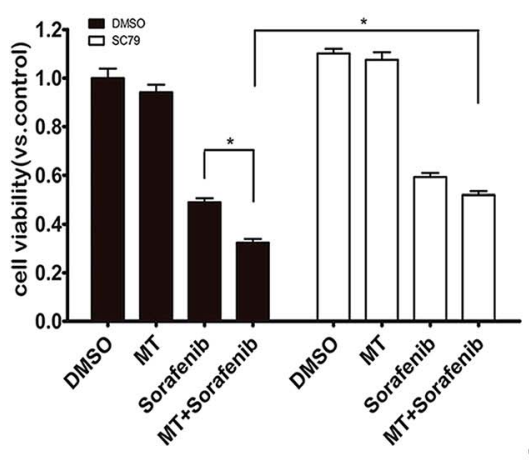

C

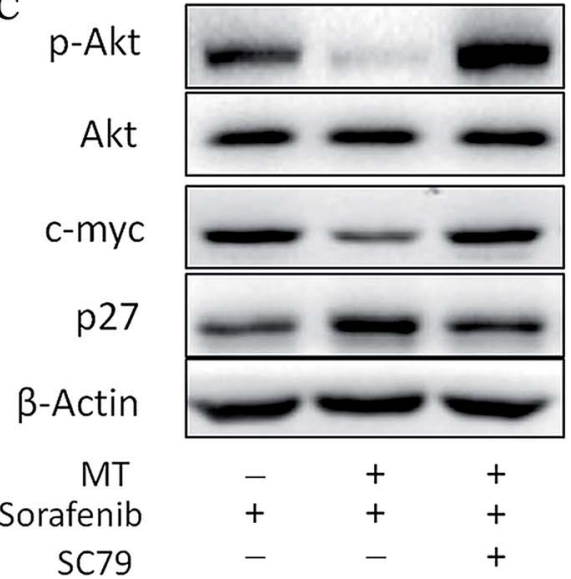

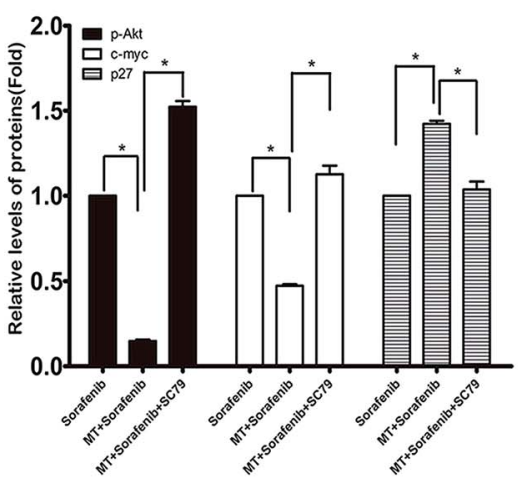

Fig. 4 Reactivation of p-AKT abrogated the G0/G1 phase arrest and the inhibitory effect on cell viability as well as the expression of relevant regulatory proteins, induced by the combined treatment of MT and sorafenib in Bel-7402 cells. Cells were treated with MT [1.0 mM] or/and sorafenib $[8.0 \mu \mathrm{M}]$ for 48 hours in the presence or absence of SC79 $\left[8.0 \mu \mathrm{g} \mathrm{ml}^{-1}\right]$ for 48 hours. (A) Cell cycle distribution in Bel-7402 cells was analysed by flow cytometry with PI staining. The percentage of different cell cycle phases was drawn in a histogram to reflect the alteration of cell cycle phases (right panel). The mean and SD obtained from three independent experiments are plotted. (B) Cell viability was measured using CCK-8 assay after 48 hours. (C) After the corresponding treatment p-AKT, AKT, p27 and c-myc protein levels were examined using western blotting. ( $P<0.05$ indicates significant difference among the groups).

\subsection{MT enhances the sorafenib-mediated inhibition of liver cancer growth in a xenograft tumor model in vivo}

To validate the enhanced sorafenib-mediated inhibition of liver cancer growth exerted by MT, we analyzed the effects of MT and sorafenib treatment in vivo using SMMC-7721 xenograft nude mouse model. Single or combined treatment of MT $25 \mathrm{mg}$ $\left.\mathrm{kg}^{-1}\right)^{34}$ and sorafenib $\left.(10 \mathrm{mg} \mathrm{kg})^{-1}\right)^{35}$ were administered for 18 days, where both the tumor volume and tumor weight were measured. As shown in Fig. 5A-C, treatment with MT and sorafenib together markedly enhanced the inhibitory effect on tumor growth compared with the sorafenib treatment alone in the xenograft model. Furthermore, no significant weight loss (Fig. 5D) or other signs of acute or delayed toxicity were observed during treatment. These data implied that combined treatment of MT and sorafenib may be a potential therapeutic approach with low toxicity for the treatment of HCC.

\section{Discussion}

Hepatocellular carcinoma (HCC) is one of the most common causes of cancer mortality worldwide. ${ }^{36,37}$ Currently, there are few effective chemotherapy drugs to combat this highly malignant cancer. ${ }^{38}$ Sorafenib, the first oral multikinase inhibitor approved for the treatment of HCC, was reported to inhibit proliferation of cancer cells and block angiogenesis. ${ }^{39,40}$ Although sorafenib showed survival benefits in large randomized phase III studies, the response rate is rather low. ${ }^{2,41}$ In addition, sorafenib has been reported to have several side effects and many patients have to use it at a reduced dose or even stop the treatment. ${ }^{42}$ In order to increase sorafenib sensitivity and reduce its associated toxicity in advanced HCC, many research groups have explored combination therapies. ${ }^{43-46} \mathrm{MT}$ exhibits an inhibition of cell proliferation, invasiveness and metastatic properties in cancer cell cultures. ${ }^{47,48}$ Indeed, MT can enhance the activity of many chemotherapeutic agents allowing the use of lower doses of these agents and thus reducing their side effects. ${ }^{49,50}$

In this study, we demonstrated not only the combined antitumor effects of MT and sorafenib against HCC in vitro, but also a novel mechanism by which MT sensitized HCC cells to sorafenib. In addition, our study evaluated the anticancer activity of MT-sorafenib combined treatment on tumor growth in 

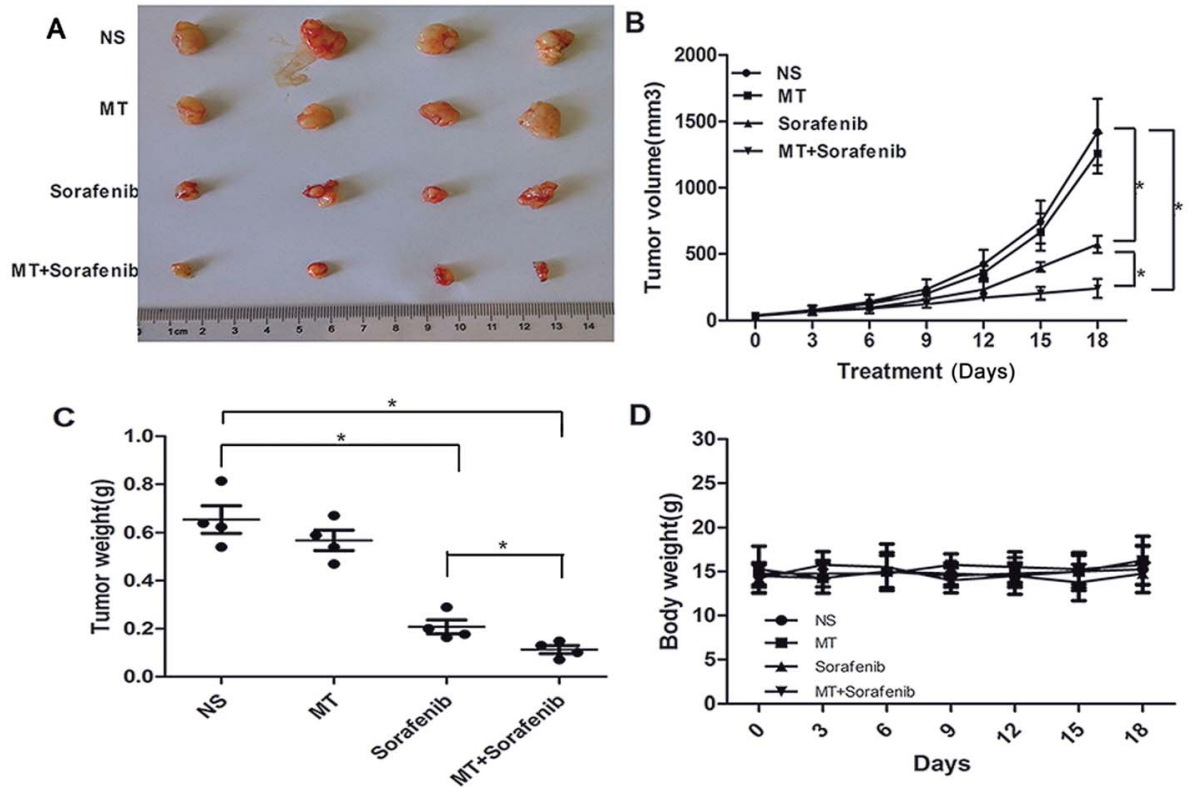

Fig. 5 MT enhanced the sorafenib-mediated inhibition of liver cancer growth in a xenograft tumor model in vivo. SMMC-7721 cells were injected subcutaneously into nude mice to evaluate the effects of MT and sorafenib. (A) Tumor pictures. (B) Tumor volumes at different times. (C) Tumor weight. (D) Body weight of mice ( $* P<0.05$ indicates significant difference among the groups. Four mice were used for each group).

xenograft-bearing nude mice. Furthermore, the cytotoxicity of the co-treatment showed no significant difference from that treated with sorafenib, consistent with previous reports. ${ }^{28}$

There are four phases in the eukaryotic cell cycle. At the G1 phase, cells begin to grow and prepare for protein synthesis, a phase related to DNA replication. Then cells enter the $\mathrm{S}$ phase when nuclear DNA is replicated. Cells continue to grow in the G2 phase preparing for mitosis (M). Finally, sister chromatids are separated and distributed to the newly forming daughter cells in the $\mathrm{M}$ phase. ${ }^{.1}$ However, the cell cycle of cancer cells is often deregulated as a result of genetic mutations, which lead to uncontrolled cell proliferation. ${ }^{52,53}$ Therefore, the inhibition of the cell cycle might be a suitable strategy for the treatment of cancer as well as other proliferative diseases..$^{54-56}$

In the present study, we examined the cell cycle and investigated the inhibition of cell proliferation. Interestingly, we found that treatment of cells with sorafenib alone induced G1/ G0 phase arrest in HCC cells, which was further induced by sorafenib and MT combination, consistent with other publications. ${ }^{57}$ These results suggest that cell arrest at the G1/G0 phase

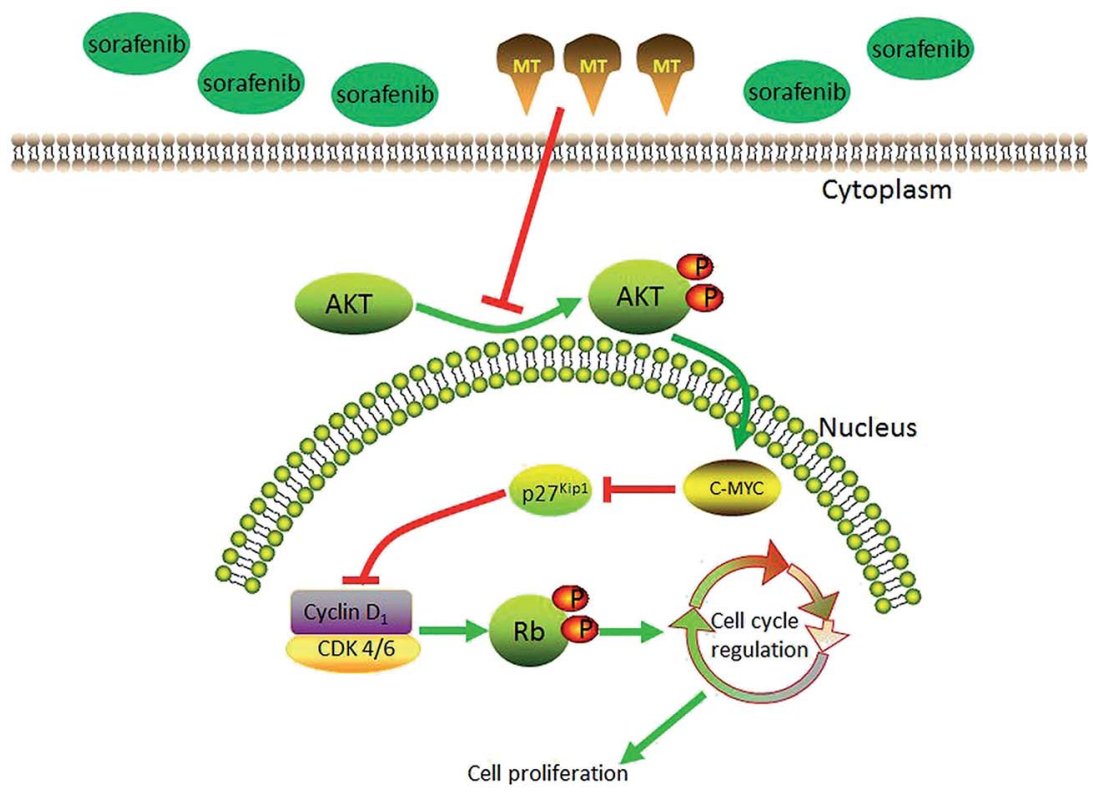

Fig. 6 Proposed model elucidating the role of MT in regulating the proliferation of hepatocellular carcinoma (HCC) cells treated with sorafenib. 
is the biochemical basis for the anticancer effect of the MTsorafenib combination.

Progression through the eukaryotic cell cycle is governed by a complex regulatory system whose central component is the cyclin-dependent kinase (CDK). CDK activation requires association with a cyclin subunit, as well as phosphorylation of the CDK subunit by the CDK-activating kinase (CAK). The three major classes of cyclins, called G1/S, S, and M cyclins, oscillate in the cell cycle to generate a series of cyclin-CDK complexes that are abruptly switched on at specific cell-cycle transitions. ${ }^{58}$ The general procedure in mammals is that CDK4 and CDK6 together with cyclin $\mathrm{D}$ initiate the phosphorylation of $\mathrm{Rb}$ proteins which can release the transcription factor E2F protein, leading to the induction of the transition from $\mathrm{G} 1$ to $\mathrm{S}$ phase. ${ }^{51}$ Next, we examined the markers related to cell cycle arrest at G1. Interestingly, we found significant differences between sorafenib treatment alone and combined treatment of sorafenib plus MT regarding the expression of cyclin D1, CDK4, CDK6, and $\mathrm{p}-\mathrm{Rb}$. The activities and effects of $\mathrm{CDK} /$ cyclin complex at different conditions including stress, DNA damage, and others, are regulated by $\mathrm{p} 27 \mathrm{Kip} 1$ which can inhibit CDK4/6/cyclin D, CDK2/cyclin E, CDK2/cyclin A, CDK1/cyclin A, as well as CDK1/ cyclin B activity. ${ }^{59,60}$ It has been reported that the effect of p27Kip1 on the activity of cyclin D-CDK4/6 is determined by the growth state of the cells..$^{61,62}$ In proliferating cells, p27 can bind to cyclin D-CDK4/6 complex without affecting its activity, whereas p27 can inactivate cyclin D-CDK4/6 complex in quiescent or resting cells. ${ }^{61}$ p27Kip1 is an inhibitor of a broad spectrum of CDKs, whose transcription and post-translation is regulated by MYC. MYC is an oncogenic transcription factor that can also suppress p27Kip1 expression through several pathways. ${ }^{32}$ MYC exerts its function at least in part through antagonizing p27 function as CDK inhibitor. ${ }^{63}$ Previous reports showed that the expression of MYC could be regulated by the AKT pathway ${ }^{64}$ Moreover, in the early G1 phase, phosphorylation at Thr157 and Thr198 by AKT caused a delay in nuclear import of p27Kip $1{ }^{33}$ which functions in the nucleus. Vanesa Martín and colleagues showed that MT inhibits AKT activity in human glioma cells without modifying MAPKs (ERK, p38, and JNK) activity. ${ }^{50}$ Bo Zhai et al. showed that inhibition of AKT reversed the acquired resistance to sorafenib. ${ }^{65}$ Therefore, we next questioned whether p-AKT could regulate p27 via c-myc, and thus affecting cell cycle progression. Consistent with the expression of these proteins, combined treatment of sorafenib and MT plus SC79, a p-AKT activator, restored a lower G0/G1 percentage of cells and cell viability, as well as the expression of c-myc, p27 and cell cycle-related proteins. This indicates that AKT can regulate c-myc, p27 and, subsequently, cell cycle. Thus AKT is a crucial factor in the enhanced antitumor effect of cotreatment of sorafenib and MT compared with sorafenib treatment alone. Also, ERK has been reported that it is related to cell proliferation $^{66}$ and MT could inhibit the expression of p-ERK in SK-OV-3 cells. ${ }^{28}$ Moreover, as a CDK inhibitor, p21 can inhibit multiple $\mathrm{CDK} /$ cyclin complexes through various ways. ${ }^{67}$ Accordingly, we examined the expressions of p-ERK and p21. To our disappointment, combination of MT and sorafenib could not change the expressions of the two proteins compared to sorafenib group (ESI Fig. S1 $\dagger$ ). Therefore, we guess that MT modulation of cell cycle seems to be cell-type and contextdependent, similar to its effects on other signaling pathways. The proposed model elucidating the role of MT in regulating the proliferation of hepatocellular carcinoma (HCC) cells treated with sorafenib is shown in Fig. 6. However, further studies need to be performed in order to investigate this interesting phenotype and pinpoint the precise molecular mechanism.

\section{Conclusions}

In summary, our results indicate that co-treatment of MT and sorafenib potentiated the antitumor activity and arrested the cell cycle via regulation of p-AKT. Therefore, our findings suggest that MT, which has demonstrated no significant toxicity to normal liver cells treated with sorafenib, can be a useful chemotherapeutic agent for the improvement of the therapeutic effect of sorafenib in liver cancer.

\section{Acknowledgements}

This work was supported by funds from the National Natural Science Foundation of China (81471755) and the clinical capability construction project for Liaoning provincial hospitals (LNCCC-B03-2014).

\section{References}

1 H. B. El-Serag and K. L. Rudolph, Gastroenterology, 2007, 132, 2557-2576.

2 J. M. Llovet, S. Ricci, V. Mazzaferro, P. Hilgard, E. Gane, J. F. Blanc, A. C. de Oliveira, A. Santoro, J. L. Raoul, A. Forner, M. Schwartz, C. Porta, S. Zeuzem, L. Bolondi, T. F. Greten, P. R. Galle, J. F. Seitz, I. Borbath, D. Haussinger, T. Giannaris, M. Shan, M. Moscovici, D. Voliotis, J. Bruix and S. I. S. Group, N. Engl. J. Med., 2008, 359, 378-390.

3 Y. H. Hwang, J. Y. Choi, S. Kim, E. S. Chung, T. Kim, S. S. Koh, B. Lee, S. H. Bae, J. Kim and Y. M. Park, Hepatol. Res., 2004, 29, 113-121.

4 M. A. Avila, C. Berasain, B. Sangro and J. Prieto, Oncogene, 2006, 25, 3866-3884.

5 M. A. Worns and P. R. Galle, Nat. Rev. Gastroenterol. Hepatol., 2014, 11, 447-452.

6 C. Berasain, Gut, 2013, 62, 1674-1675.

7 C. Zavaglia, A. Airoldi, A. Mancuso, M. Vangeli, R. Viganò, G. Cordone, M. Gentiluomo and L. S. Belli, Eur. J. Gastroenterol. Hepatol., 2013, 25, 180-186.

8 R. J. Reiter, Endocr. Rev., 1991, 12, 151-180.

9 J. Vanecek, Physiol. Rev., 1998, 78, 687-721.

10 D. X. Tan, L. C. Manchester, L. Fuentes-Broto, S. D. Paredes and R. J. Reiter, Obes. Rev., 2011, 12, 167-188.

11 A. Carrillo-Vico, J. M. Guerrero, P. J. Lardone and R. J. Reiter, Endocrine, 2005, 27, 189-200.

12 E. Mills, P. Wu, D. Seely and G. Guyatt, J. Pineal Res., 2005, 39, 360-366. 
13 B. Jung-Hynes, R. J. Reiter and N. Ahmad, J. Pineal Res., 2010, 48, 9-19.

14 S. Y. Shiu, B. Pang, C. W. Tam and K. M. Yao, J. Pineal Res., 2010, 49, 301-311.

15 P. T. Ram, J. Dai, L. Yuan, C. Dong, T. L. Kiefer, L. Lai and S. M. Hill, Cancer Lett., 2002, 179, 141-150.

16 L. Mao, Q. Cheng, B. Guardiola-Lemaitre, C. Schuster-Klein, C. Dong, L. Lai and S. M. Hill, J. Pineal Res., 2010, 49, 210221.

17 O. Trubiani, R. Recchioni, F. Moroni, J. Pizzicannella, S. Caputi and R. Di Primio, J. Pineal Res., 2005, 39, 425431.

18 S. Rubio, F. Estevez, J. Cabrera, R. J. Reiter, J. Loro and J. Quintana, J. Pineal Res., 2007, 42, 131-138.

19 G. Garcia-Santos, I. Antolin, F. Herrera, V. Martin, J. Rodriguez-Blanco, M. del Pilar Carrera and C. Rodriguez, J. Pineal Res., 2006, 41, 130-135.

20 G. Z. Omurtag, A. Tozan, A. O. Sehirli and G. Sener, J. Pineal Res., 2008, 44, 432-438.

21 M. Valko, H. Morris and M. T. Cronin, Curr. Med. Chem., 2005, 12, 1161-1208.

22 R. J. Reiter, D.-X. Tan, R. M. Sainz, J. C. Mayo and S. LopezBurillo, J. Pharm. Pharmacol., 2002, 54, 1299-1321.

23 E. Esposito, A. Iacono, C. Muia, C. Crisafulli, G. Mattace Raso, P. Bramanti, R. Meli and S. Cuzzocrea, J. Pineal Res., 2008, 44, 78-87.

24 F. Herrera, V. Martin, G. Garcia-Santos, J. Rodriguez-Blanco, I. Antolin and C. Rodriguez, J. Neurochem., 2007, 100, 736746.

25 E. Peschke, J. Pineal Res., 2008, 44, 26-40.

26 P. Lissoni, S. Barni, M. Mandala, A. Ardizzoia, F. Paolorossi, M. Vaghi, R. Longarini, F. Malugani and G. Tancini, Eur. J. Cancer, 1999, 35, 1688-1692.

27 S. Klongpanichapak, P. Phansuwan-Pujito, M. Ebadi and P. Govitrapong, J. Pineal Res., 2007, 43, 65-73.

28 J. H. Kim, S. J. Jeong, B. Kim, S. M. Yun, D. Y. Choi and S. H. Kim, J. Pineal Res., 2012, 52, 244-252.

29 Z. A. Stewart, M. D. Westfall and J. A. Pietenpol, Trends Pharmacol. Sci., 2003, 24, 139-145.

30 J. A. Pietenpol, S. K. Bohlander, Y. Sato, N. Papadopoulos, B. Liu, C. Friedman, B. J. Trask, J. M. Roberts, K. W. Kinzler, J. D. Rowley, et al., Cancer Res., 1995, 55, 1206-1210.

31 K. Polyak, J. Y. Kato, M. J. Solomon, C. J. Sherr, J. Massague, J. M. Roberts and A. Koff, Genes Dev., 1994, 8, 9-22.

32 S. S. Hnit, C. Xie, M. Yao, J. Holst, A. Bensoussan, P. De Souza, Z. Li and Q. Dong, Int. J. Biochem. Cell Biol., 2015, 68, 9-14.

33 D. Morishita, R. Katayama, K. Sekimizu, T. Tsuruo and N. Fujita, Cancer Res., 2008, 68, 5076-5085.

34 J. J. Lu, L. Fu, Z. Tang, C. Zhang, L. Qin, J. Wang, Z. Yu, D. Shi, X. Xiao, F. Xie, W. Huang and W. Deng, Oncotarget, 2016, 7, 2985-3001.

35 Y. Liang, T. Zheng, R. Song, J. Wang, D. Yin, L. Wang, H. Liu, L. Tian, X. Fang, X. Meng, H. Jiang, J. Liu and L. Liu, Hepatology, 2013, 57, 1847-1857.
36 S. Waly Raphael, Z. Yangde and C. Yuxiang, ISRN Oncol., 2012, 2012, 421673.

37 A. Forner, J. M. Llovet and J. Bruix, Lancet, 2012, 379, 12451255.

38 R. Cabrera and D. R. Nelson, Aliment. Pharmacol. Ther., 2010, 31, 461-476.

39 A. Galmiche, B. Chauffert and J. C. Barbare, Cancer Lett., 2014, 346, 159-162.

40 V. Di Marco, F. De Vita, J. Koskinas, D. Semela, P. Toniutto and C. Verslype, Ann. Oncol., 2013, 24(2), 30-37.

41 A. L. Cheng, Y. K. Kang, Z. Chen, C. J. Tsao, S. Qin, J. S. Kim, R. Luo, J. Feng, S. Ye, T. S. Yang, J. Xu, Y. Sun, H. Liang, J. Liu, J. Wang, W. Y. Tak, H. Pan, K. Burock, J. Zou, D. Voliotis and Z. Guan, Lancet Oncol., 2009, 10, 25-34.

42 J. T. Hartmann, M. Haap, H. G. Kopp and H. P. Lipp, Curr. Drug Metab., 2009, 10, 470-481.

43 B. Zhai and X. Y. Sun, World J. Hepatol., 2013, 5, 345-352.

44 C. H. Hsu, Y. C. Shen, Z. Z. Lin, P. J. Chen, Y. Y. Shao, Y. H. Ding, C. Hsu and A. L. Cheng, J. Hepatol., 2010, 53, 126-131.

45 A. Y. Chang and M. Wang, Anti-Cancer Drugs, 2013, 24, 251259.

46 C. Hsu, Y. C. Shen and A. L. Cheng, Expert Rev. Clin. Pharmacol., 2009, 2, 129-136.

47 S. Cos, R. Fernandez, A. Guezmes and E. J. Sanchez-Barcelo, Cancer Res., 1998, 58, 4383-4390.

48 M. M. Marelli, P. Limonta, R. Maggi, M. Motta and R. M. Moretti, Prostate, 2000, 45, 238-244.

49 S. Casado-Zapico, J. Rodriguez-Blanco, G. Garcia-Santos, V. Martin, A. M. Sanchez-Sanchez, I. Antolin and C. Rodriguez, J. Pineal Res., 2010, 48, 72-80.

50 V. Martín, G. García-Santos, J. Rodriguez-Blanco, S. CasadoZapico, A. Sanchez-Sanchez, I. Antolín, M. Medina and C. Rodriguez, Cancer Lett., 2010, 287, 216-223.

51 H. Harashima, N. Dissmeyer and A. Schnittger, Trends Cell Biol., 2013, 23, 345-356.

52 G. I. Evan and K. H. Vousden, Nature, 2001, 411, 342-348.

53 L. H. Hartwell and M. B. Kastan, Science, 1994, 266, 18211828.

54 K. W. Kohn, J. Jackman and P. M. O'Connor, J. Cell. Biochem., 1994, 54, 440-452.

55 B. T. Hill and R. Baserga, Cancer Treat. Rev., 1975, 2, 159175.

56 W. J. Chen and J. K. Lin, J. Biol. Chem., 2004, 279, 1349613505.

57 Q. Cao, Y. Jiang, J. Shi, X. Liu, J. Chen, T. Niu and X. Li, Clin. Exp. Pharmacol. Physiol., 2015, 42, 502-509.

58 D. O. Morgan, Cell, 2008, 135, 764.

59 H. Toyoshima and T. Hunter, Cell, 1994, 78, 67-74.

60 O. Aprelikova, Y. Xiong and E. T. Liu, J. Biol. Chem., 1995, 270, 18195-18197.

61 M. K. James, A. Ray, D. Leznova and S. W. Blain, Mol. Cell. Biol., 2008, 28, 498-510.

62 A. Ray, M. K. James, S. Larochelle, R. P. Fisher and S. W. Blain, Mol. Cell. Biol., 2009, 29, 986-999.

63 G. Bretones, M. D. Delgado and J. Leon, Biochim. Biophys. Acta, 2015, 1849, 506-516. 
64 M. A. Dominguez-Caceres, J. M. Garcia-Martinez, A. Calcabrini, L. Gonzalez, P. G. Porque, J. Leon and J. Martin-Perez, Oncogene, 2004, 23, 7378-7390.

65 B. Zhai, F. Hu, X. Jiang, J. Xu, D. Zhao, B. Liu, S. Pan, X. Dong, G. Tan, Z. Wei, H. Qiao, H. Jiang and X. Sun, Mol. Cancer Ther., 2014, 13, 1589-1598.
66 M. Cully and J. Downward, Cell, 2008, 133, 1292.

67 Y. Wang, J. C. Fisher, R. Mathew, L. Ou, S. Otieno, J. Sublet, L. Xiao, J. Chen, M. F. Roussel and R. W. Kriwacki, Nat. Chem. Biol., 2011, 7, 214-221. 